\title{
VKHUTEMAS y su papel en el diseño posrevolucionario Soviético
}

\author{
VKHUTEMAS and its role in the Soviet post-revolutionary design \\ Viktoriya Kondratenko Mozgova \\ ${ }^{1}$ Departamento de Historia del Arte, Universidad de Málaga, España (kondratenko.victoria@ gmail.com)
}

Recibido el 28 de marzo de 2017; revisado el 10 de abril de 2017; aceptado el 15 de abril de 2017 de 2017; publicado el 01 de julio de 2017

RESUMEN: Es difícil definir la importancia que supuso VKHUTEMAS en la historia del diseño soviético, ya que fue toda una revolución en el ámbito artístico del país, a modo de la Revolución de Octubre. No solo fue la creación de la primera escuela de diseño en el este de Europa, sino la aparición de un tipo de profesionales altamente cualificados, capaces de romper con la tradición y realizar un nuevo tipo de arte. VKHUTEMAS, al igual que la Bauhaus, se convirtió en el centro de un nuevo lenguaje y el motor principal para el potencial de una nueva generación. En este trabajo se busca hacer un breve acercamiento a la creación de un nuevo sistema y una nueva metodología totalmente diferente a las conocidas en aquel entonces, en un país que en los primeros años del siglo pasado nada tenía que envidiar a los grandes núcleos artísticos europeos.

PALABRAS CLAVE: Vkhutemas, diseño, años 20, URSS, enseñanza artística.

\begin{abstract}
It's hard to describe the relevance of the influence that the VKHUTEMAS had had in the history of soviet design, for it had taken its country to one of the most important revolutions since the October Revolution. Besides the creation of the first school of design in the East of Europe, it gave the possibility for highly skilled professionals to make their appearance, who had been capable of breaking with tradition and of creating a new kind of art. VKHUTEMAS just like the Bauhaus, became the heart of a new language and the driving force of the potential of a new generation. The main goal of this Project is to offer a brief explanation of the creation of a new system and a new methodic, which were completely variant to the ones known at the time in this country, that had no reason to feel jealous of the great European artistic core in the first years of the last century.
\end{abstract}

KEYWORDS: Vkhutemas, design, the 1920s, URSS, artistic education. 
Después de la tercera reforma educativa y la disolución de Vysshiy Gosudarstvennyi Khudozhestvennotekhnicheskiy Institut (en adelante VKHUTEIN), su huella fue prácticamente borrada: la historia de la existencia olvidada y los materiales artísticos del archivo - destruidos o guardados en colecciones privadas-. La única excepción fue la parte de documentación, aunque dejada en la sombra durante muchos años. Años en los que no se le otorgaba importancia a una cuna artística tan importante para el desarrollo artístico del Este como lo fue Bauhaus en el Occidente. En su momento fue una revolución, una transformación plástica de la gran unión de países, un nuevo estilo de vida y un nuevo arte. El primer trabajo acerca del tema y el que supo demostrar el valor de esta institución y este momento artístico fue la recuperación de la memoria de Vysshie Gosudarstvennye Khudozhestvennotekhnicheskie Masterskie (en adelante VKHUTEMAS) realizada por S.O. Khan-Magomedov, aquien se deben casi todos los estudios posteriores.

\section{VKHUTEMAS: los primeros pasos hacia el nuevo arte}

VKHUTEMAS se formó en un momento muy especial en la historia de Rusia: justo tras la Revolución rusa de 1917, la cual tomó relevo a la Gran Guerra, constituyendo, así, los primeros años del gobierno soviético. Para entender a aquellos jóvenes artistas que vivían de las ideas románticas de transformar el mundo, habría que meterse en el espíritu de la época. Fue un período de crisis y grandes cambios. Podemos hablar de crisis económica, social, política y también de las ideológicas, ya que, tras la agitación y elocuencia social, llegó un período de revolución espiritual. Por ello, el cambio en el mundo artístico no se puede explicar solamente a través del concepto de vanguardia. No se trata de romper el sistema, ni tampoco de "camuflar lo antiguo", sino de cambiarlo desde dentro, creando un nuevo lenguaje que responda a las necesidades y peticiones de la nueva sociedad, y transformando la profesión del artista desde su raíz, estableciendo, por lo tanto, unas relaciones totalmente distintas entre el artista y el arte.

La tradición del diseño industrial en Rusia se remonta al siglo XIX. En 1825, en Moscú, fue inaugurada la academia de dibujo en relación con las artes y oficios de Stroganov -Moskovskaya gosudarstvennaya khudozhestvenno-promyshlennaya akademiya im. Stroganova (en adelante MGKHPA)-. Otra institución importante en la formación de VKHUTEMAS fue la Escuela de Pintura, Escultura y Arquitectura de Moscú - Moskovskoe Uchilishche Zhivopisi, Vayaniya i Zodchestva (en adelante MUZHVZ)-, contemporánea a la mencionada anteriormente. La principal diferencia entre las dos instituciones artísticas más importantes de la época fue un frente abierto entre sus representantes: los seguidores de MUZHVZ defendían el arte puro y los del MGHPA el arte aplicado. El 
enfrentamiento entre los puristas y los productivistas seguirá existiendo incluso después de la abolición de las dos instituciones, trasladándose a una nueva, VKHUTEMAS, a pesar de todos los esfuerzos por erradicar el conflicto.

Con la caída de la Academia de Arte como institución principal aparecieron muchas asociaciones y comités que buscaban formar a artistas en un ámbito interdisciplinar y orientarlos a la producción industrial, capaces de enlazar lo artístico con una nueva visión de la sociedad. Esto llevará a una primera reforma artística y a la aparición de una nueva enseñanza que reunía el concepto de relación maestro-alumno de carácter renacentista. Tras la reorganización del sistema educativo artístico, las dos escuelas más importantes de Moscú fueron abolidas y en su lugar, en 1918, se crearon los Primeros y Segundos Talleres Libres del Estado, cuya idea principal era separarse de la enseñanza tradicional e implantar un nuevo sistema subjetivo de talleres individuales, dando completa libertad a los oficios. Hay que remarcar que el objetivo principal no era la búsqueda de una escuela de diseño, sino el deseo de "formar al pueblo en las bellas artes para que tenga una completa percepción de la cultura artística en los campos de la pintura, la arquitectura y la escultura" (Lodder, 1988: 111). De esta manera se democratizó el arte, abriendo las puertas de la enseñanza a todas las clases sociales, independientemente de su estatus y calificaciones anteriores. La creación de unos talleres libres como su propio nombre indica, otorgaba a los jóvenes una completa libertad a la hora de aprender y crear. Por ello, en cuanto a las asignaturas impartidas, se intentó abarcar todas las corrientes artísticas existentes en aquellos años, desde las más tradicionales hasta las más vanguardistas y, por otro lado, cada alumno podía elegir libremente a su maestro - y por ende la enseñanza que más le guste - o, incluso, estudiar sin maestro, como fue el caso de Obschestvo molodyh khudozhnikov, OBMOKHU (Sociedad de Artistas Jóvenes). Además, pese a asemejarse al sistema renacentista, los estudiantes podían cambiarse de taller cuando quisieran, "un poder que influía negativamente en el número de adeptos de los maestros más innovadores como Tatlin o Malevich, pero que permitía al estudiante realizarse como artista sin ningún tipo de normas impuestas por la dirección” (Mikhaylov, 2002: 261).

Los primeros dos años, los Talleres funcionaron sin un programa establecido, siendo fieles a la idea de completa libertad de los estudiantes. No obstante, ya durante el primer año de estudio, sobre la base del nuevo sistema aparecieron una serie de problemas. El principal consistía en la subjetividad del método. Los estudiantes no recibían la formación artística completa, sino solo el modelo heredado de su maestro. Tal situación creó gran descontento, no solo entre los estudiantes, sino también entre algunos profesores, quienes proponían buscar un sistema más objetivo con el cual defender sus experimentos artísticos (Mikhaylov, 2002: 260). Por lo tanto, estaba muy claro que tan solo con 
separarse del sistema académico anterior no bastaba, sino que también hacía falta proyectar y ejecutar un sistema pedagógico específico.

Ante el problema de la diversidad de tendencias y la falta de un programa pedagógico para las mismas, apareció la necesidad de una nueva reforma que pudiera organizar la institución y apaciguar los conflictos internos1. Por lo tanto, en 1920 se llevó a cabo una segunda reforma educativa, en la cual, los dos Talleres Libres Mayores se unieron en una única estructura a la que nombraron VKHUTEMAS, cuyo decreto fundacional la definía como (Colón, 2002: 127):

\begin{abstract}
"un establecimiento superior de enseñanza artística especializada, teniendo por objeto la preparación de maestros-obreros, artistas de calificación superior para la industria, así como de instructores y dirigentes para la formación técnica profesional"
\end{abstract}

VKHUTEMAS reflejó toda la complejidad del proceso artístico ruso de los primeros años del siglo pasado. De su núcleo salieron muchas otras asociaciones como Assotsiatsiya Novykh Arkhitektorov (ASNOVA), Obschestvo Khudozhnikov-Stankovistov (OST), Ob'edinenie Svobodnyh Arkhitektorov (OSA), Assotsiatsiya Arkhitektorov-Urbanistov (ARU), entre otras, formadas tanto por los antiguos alumnos de Vkhutemas como por los profesores del mismo. En otras palabras, podemos hablar de una gran estructura dentro de la cual convivían muchas tendencias, a veces rivales entre ellas y en las cuales, el papel fundamental lo tenían los estudiantes, quienes, con mucho entusiasmo, participaban en la vida de la escuela e influían en su orientación casi al mismo nivel de sus profesores. Esa libertad en la actividad y en la creación artística en sí aumentaba el potencial artístico de la escuela como generadora y catalizadora de ideas innovadoras. Por ello, tampoco podemos hablar de una estructura estática, sino una institución dinámica, en continuo movimiento y cambio. Su estructura y programa se modificaban conforme a los cambios tanto a nivel político, como a nivel interno (sucesión de rectores)2.

A pesar de su naturaleza dinámica, en 1923 se estableció un único programa que se estructuraba desde el aprendizaje pictórico-plástico básico hasta el específico profesional. La primera parte se ocupaba de proporcionar los principios artísticos en un curso básico impartido a todos los estudiantes

\footnotetext{
${ }^{1}$ A los anteriormente mencionados puristas (pintores de caballete como Shevchenko, Lentulov, Fedorov, Falk, etc.) y los de las artes aplicadas (Favorski, Novinski, Egorov, etc.) se sumaron los constructivistas y productivistas (Rodchenko, Popova, Lavinski, Vesnin, etc.) que cada vez ganaban más popularidad y luchaban por conquistar una posición dominante.

2 Toda la historia de la escuela se puede dividir en tres importantes periodos que corresponden a los tres rectorados: Ravdel (1920-1923), Favorskiy (1923-1926), Novitskiy (1926-1930). Esto es muy importante, ya que tanto la estructura como el profesorado de VKHUTEMAS se modificaban según el rector que había al mando, en gran parte debido al antigüo conflícto entre los puristas y productivistas.
} 
por igual, y la segunda ofrecía una formación específica dependiendo de la facultad que escogía el alumno. De esta manera, el fundamento artístico era igual para todos los artistas formados en VKHUTEMAS.

Como hemos visto, los talleres abrieron sus puertas a toda clase de jóvenes, sin tener en cuenta sus anteriores calificaciones o preparación específica, pero esta idea tan romántica dejó al descubierto las diferencias existentes entre los estudiantes. Por lo tanto, había que crear un curso que igualara el nivel de los alumnos, y este fue Rabochiy fakultet (Rabfak), cuyo objetivo no era otro que preparar a los jóvenes para entrar en VKHUTEMAS. Allí, durante cuatro años aprendían conceptos requeridos para el posterior curso básico, empezando desde lo más esencial hasta las nociones de la construcción y composición.

\section{VKHUTEMAS y Bauhaus}

Antes de avanzar, tracemos un paralelismo entre la división de la enseñanza en VKHUTEMAS y Bauhaus [1 y 2]. Su estructura es muy parecida: ambas comparten el concepto del célebre vorkurs (enseñanza preliminar); un segundo ciclo que proporcionaba al estudiante la práctica necesaria para el desarrollo de su actividad artística, que corresponde a los distintos talleres de VKHUTEMAS; y un tercero en el que los estudiantes de ambas instituciones entraban en contacto con el mundo laboral a la vez que preparaban su proyecto final.

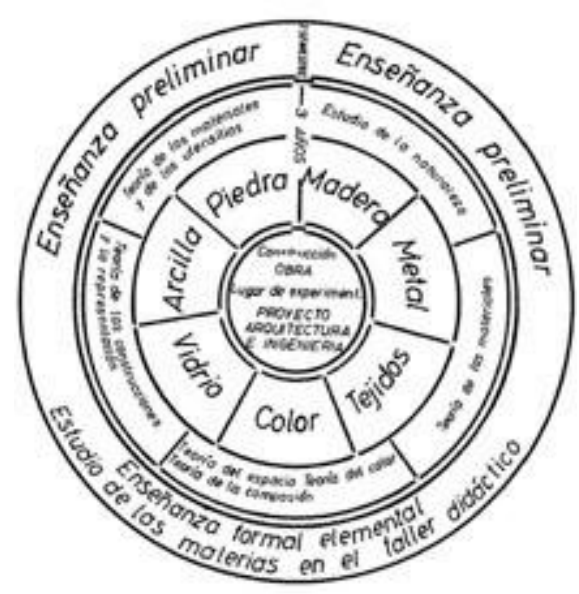

[Fig.1] Esquema circular de la enseñanza en Bauhaus. Fuente: http://teoriauno.tripod.com/documentos/Bauhaus.pdf 


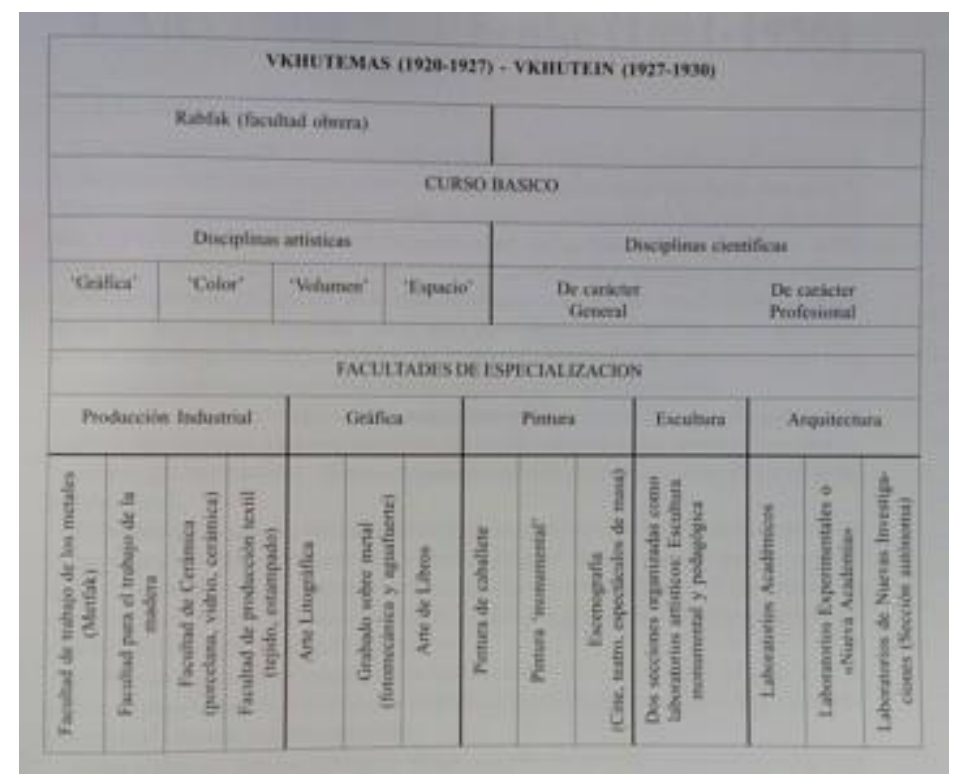

[Fig.2] Esquema de la enseñanza en VKHUTEMAS, de Colón Llamas. En: Las vanguardias artísticas y la enseñanza en la Rusia de los años 20.

Por lo tanto, después de cuatro años de Rabfak, los estudiantes de nuevo ingreso de VKHUTEMAS se enfrentaban a un sistema pedagógico que también comprendía un curso básico de preparación antes de las diferentes especializaciones - un análogo de vorkurs-, compuesto por dos grupos de asignaturas, agrupadas según su naturaleza:

1) Disciplinas científicas: física, química, matemáticas avanzadas, mecánica teórica, geometría, perspectiva, historia del arte, geografía descriptiva, etc.

2) Disciplinas artísticas (pictórico-prácticas): agrupadas en cuatro grandes bloques (gráfica, color, volumen y espacio).

De las obras pertenecientes a este curso, lo primero que llama la atención es el carácter analítico de la misma, tan propio de la época y del contexto artístico europeo. Se sabe que en los primeros años del siglo XX los vanguardistas rusos no estaban aislados del Occidente y, por lo tanto, las principales corrientes artísticas que influyeron en las obras de los jóvenes artistas eran cubismo, fauvismo, expresionismo y algunos primeros apuntes del arte abstracto. Todos ellos buscaban la limpieza de las líneas, formas y color. Por ello, al mirar la obra de Rodchenko o Popova, podemos ver qué tipo de ejercicios realizaban los alumnos de VKHUTEMAS en la disciplina propedéutica "color": en la exposición de los trabajos de 1925 [3] hablamos de campos de color dentro de figuras geométricas 
sencillas, jugando con las diferentes tonalidades de colores puros y tonos vibrantes como el azul, verde o amarillo.

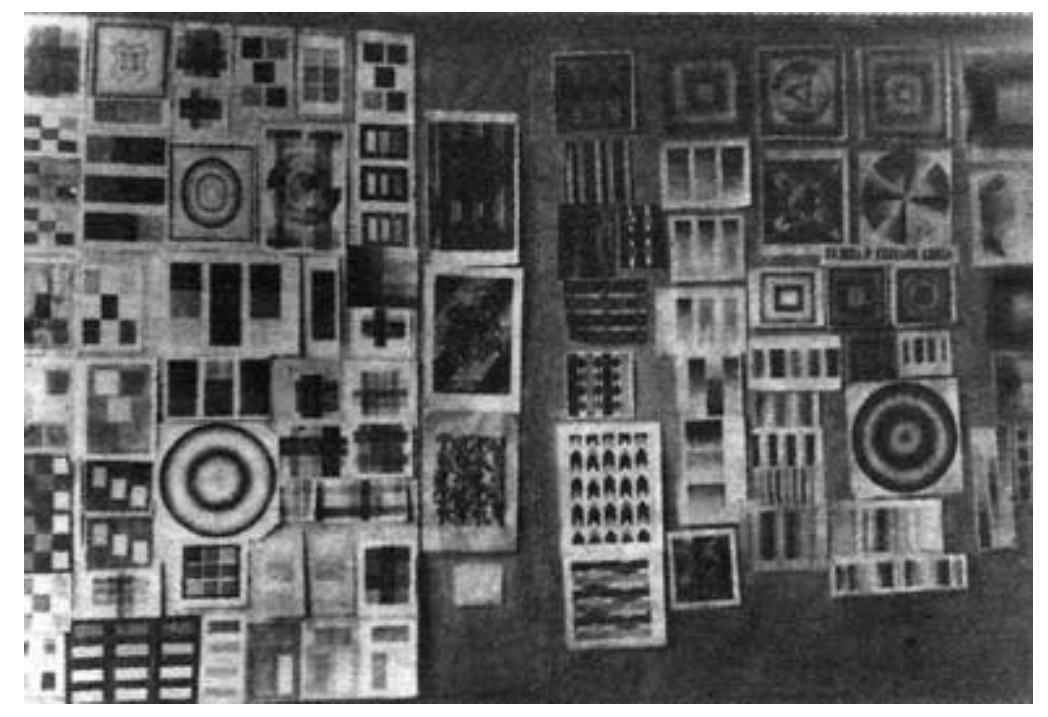

[Fig.3] Exposición de los trabajos de la disciplina Color del curso básico de VKHUTEMAS, 1925. Fuente:

(http://www.alyoshin.ru/Files/publika/khan_archi/khan_archi_1_040.html)

En cuanto a las especializaciones que ofertaba la institución, estas se dividían en cuatro grupos, y cada uno, a su vez, se componía de otras varias. De esta manera hablamos de cuatro grupos u ocho facultades: Producción Industrial -que a su vez se dividía en Trabajo de los metales (Metalloobrabatyvayuschiy fakultet - Metfak), Trabajo de la madera (Derevoobrabatyvayuschiy fakultet - Derfak), Cerámica (Keramicheskiy fakultet - Kerfak) y Producción textil (Tekstil'nyi fakultet -Tekstfak)-; Gráfica; Pintura; Escultura y Arquitectura. El estatus de todas las facultades era igualitario, aunque no se puede decir lo mismo de su posterior importancia en la historia del diseño del país, ya que con el tiempo destacaron sobre las demás las siguientes facultades: Arkhfak, Derfak, Metfak y la posterior unión de estas dos últimas. En ellas, los profesionales "ingenieros-pintores" trabajaban para la precisión del nuevo perfil creado y el desarrollo del papel del diseñador en la formación de esta esfera de creación. En la Facultad de los Metales se realizaban diseños tan interesantes como la mesa plegable del estudiante Morozov [4 y 5]: 

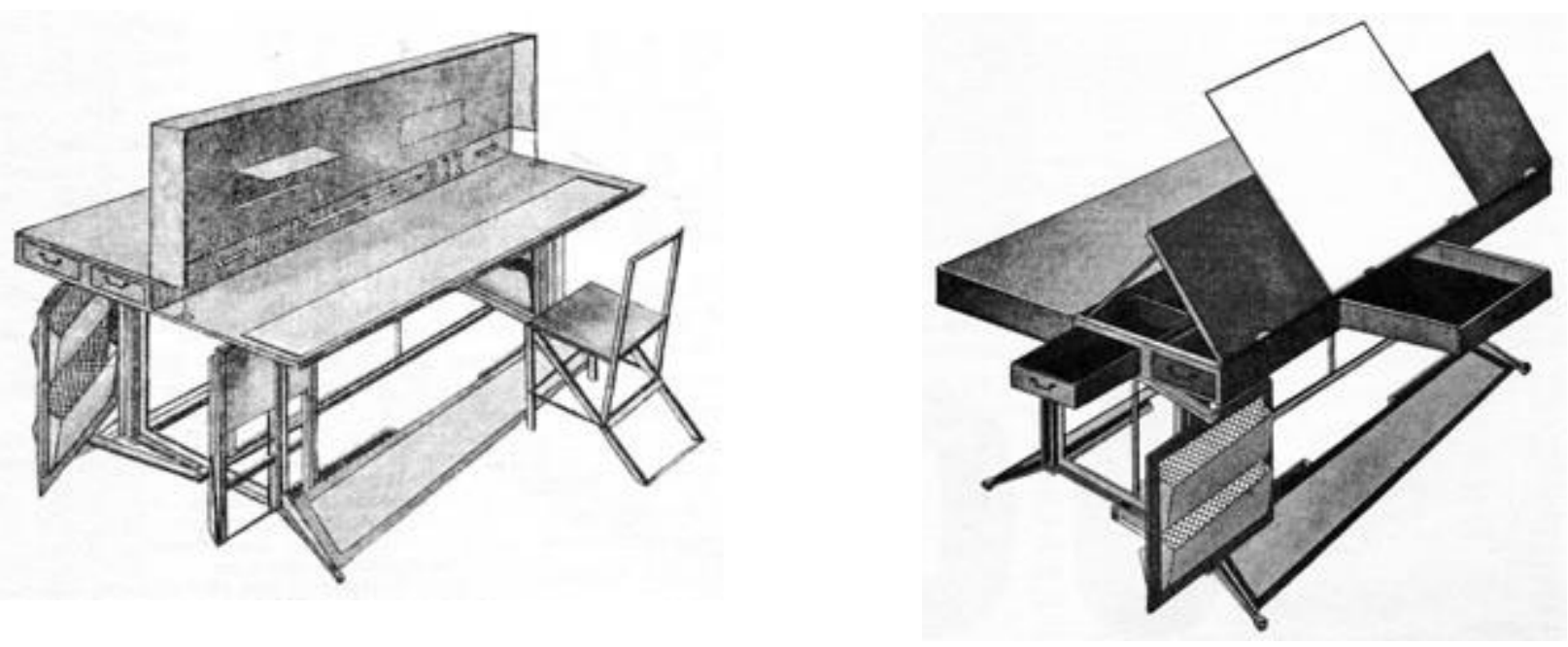

[Fig.4 y 5]. Proyecto del constructivista Morozov, Moscú, VKHUTEMAS, 1926. Fuente: http://tehne.com/event/arhivsyachina/arhiv-konstruktivizm-v-armature-povsednevnogo-byta-1926

Se trata de una mesa universal, construcción que combina varios objetos que la hacen útil, tanto para comer como para realizar trabajos técnicos. El tablero está dividido en dos partes y una de ellas se levanta perpendicularmente, dejando al descubierto los aros y elementos de sujeción para los cubiertos y vajillas, así como un rollo de papel que sirve como mantel. Esta parte del tablero, cuando no se utiliza como mesa de comer, se dobla, escondiendo la vajilla sin tocarla y su reverso puede ser utilizado para el trabajo. La otra parte también es móvil y puede fijarse bajo cualquier ángulo, sirviendo como pupitre para el dibujo técnico. Debajo de ella había una serie de cajones donde el artista podía guardar sus trabajos y sus utensilios. En la parte inferior de la mesa también había una especie de caja para guardar las revistas o planos. Las patas de la mesa están colocadas sobre ruedas, con el fin de mejorar la transportación de la misma. De esta manera puede cambiarse de sitio muy fácilmente, además de desmontarse por completo. Y, por último, su tamaño permite albergar cuatro sillas plegables.

Por su parte, uno de los ejemplos más completos e interesantes realizados por la Facultad de la Madera es la propuesta del diseño del interior constuctivista de Ródchenko, presentado en la Exposición Internacional de Artes Decorativas e Industriales Modernas de París, en 1925, y llamado El Club de Trabajadores (Lodder, 1988: 156) [6]: 


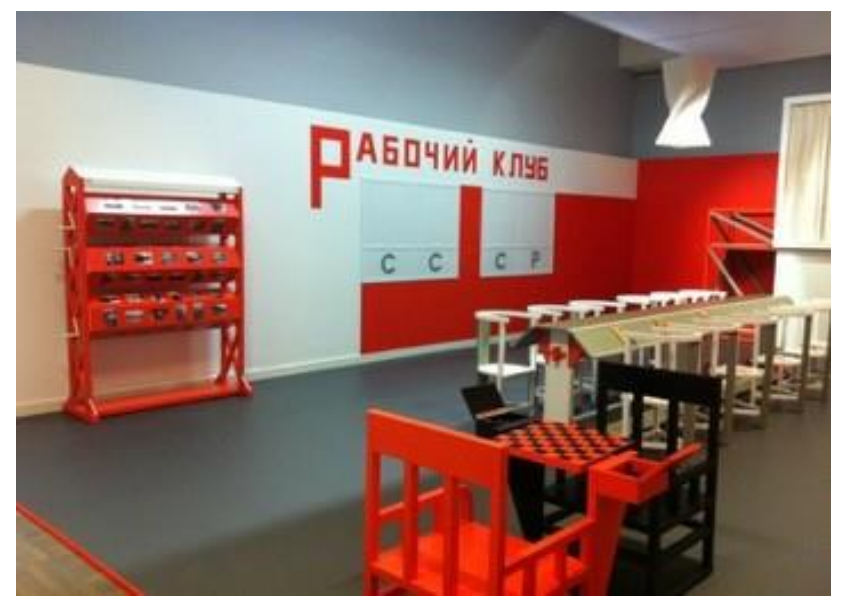

[Fig.6]. Rodchenko - El Club de los Trabajadores. Fuente: (http://lecs-ni.tumblr.com/post/81842694114)

En primer lugar, el Club servía como un espacio para la lectura, por lo tanto, había: mesa, sillas, vitrinas expositoras y armarios para el almacenamiento de libros y revistas; vitrinas móviles para los carteles, mapas, etc. También se habían preparado espacios especiales (rincón de Lenin) para las exposiciones de distintos documentos políticos con expositores móviles y plegables. Desde este punto de vista, también se necesitaba una instalación para el desarrollo de los distintos mítines y reuniones que incluían: "una tribuna para el orador, el sitio para el director o redactor del periódico, una pantalla deslizante para la presentación del material ilustrativo, pantalla móvil para las consignas y diapositivas. Toda ella plegable y fácilmente transportable de un sitio a otro" (Varst, 1926: 36). Por último, el Club de los Trabajadores, como ya se ha mencionado, era un lugar de ocio, por lo tanto, estaba dotado de muebles como la mesa con dos sillas para el juego de ajedrez.

La historia de VKHUTEMAS fue breve pero intensa, a la vez que muy dinámica, gracias a los constantes cambios. En 1926 fue pasó de nombrarse "talleres" a "instituto", pasando a ser Instituto de Enseñanza Superior del Arte y de la Técnica (VKHUTEIN). No obstante, a pesar de toda la importancia en el diseño posrevolucionaro, cuatro años después, en 1930, fue disuelto y sus facultades pasaron a formar parte de otras instituciones y universidades. VKHUTEMAS-VKHUTEIN dejó de existir, pero su huella es imborrable de la historia artística soviética. 


\section{Conclusiones}

A lo largo de los años y a través de diversas investigaciones se puede comprobar cómo, el papel de VKHUTEMAS-VKHUTEIN en la historia del diseño, cobra más y más importancia, seduciendo a los estudiosos, tanto desde su perspectiva pedagógica como desde el punto de vista institucional. Es importante tomarla como punto de referencia al hablar del nacimiento de la profesión de diseñador, así como remarcar su estatus como una escuela que nada tuvo que envidiar a la Bauhaus y que se convirtió en el centro del núcleo artístico de muchas asociaciones posteriores y punto de partida de tantos otros colectivos de artistas.

Actualmente, los documentos y materiales que se han podido conservar constituyen una importante herencia para que los alumnos de diversas facultades, como MARKHI, puedan inspirarse a través de su estudio. Por otra parte, también supone una fuente de estudio para los especialistas que analizan el sistema y la metódica de estudio, con el fin de mejorar la enseñanza pedagógica de hoy en día, destacando los logros propedéuticos de VKHUTEMAS.

No obstante, fuera del territorio ruso hay una gran falta de información sobre esta escuela (tenemos mucho más conocimiento y estudios sobre la Bauhaus), a pesar de que es sumamente importante e interesante mostrar el nacimiento de una ideología nueva y cómo se plasmó en la práctica en unos talleres que formaban una nueva especie de artistas, los artistas-artesanos, un concepto innovador, ya que hunde raíces en la tradición medieval del taller, pero conserva la idea moderna de exaltar el nombre del artista y su individualidad.

Por lo tanto, todas las investigaciones que den a conocer el nombre de VKHUTEMAS son importantes y de sumo interés. Este trabajo pretende abrir una pequeña ventana para los futuros investigadores hacia el estudio de algo tan importante como la creación de una escuela de diseño en la Rusia posrevolucionaria. Además de elaborar un estudio referente a la parte estética de los Talleres ArtísticoTécnicos Superiores del Estado, también se busca realizar un breve acercamiento al contexto cultural y al nuevo tipo de enseñanza que ofrecía Rusia a principios del siglo pasado con sus reformas más importantes y la creación de nuevas organizaciones vanguardistas, así como que mostrar la manera en la que nació la profesión del diseñador en la URSS y reconocer a los artistas más relevantes, con sus aportaciones dentro y fuera de la escuela. 


\section{Referencias bibliográficas}

ABRAMOVA, Anastasiya. (1964), "Nasledie VKHUTEMASa" en VVAA, Dekorativnoe iskusstvo SSSR, Nauka, Moscú, pp. 21-30.

BONSIEPE, Gui, (1978), Teoría y práctica del diseño industrial: elementos para una manualística crítica, Gustavo Gili D.L., Barcelona.

COLON LLAMAS, Luis Carlos, (2002), Las vanguardias artísticas y la enseñanza en la Rusia de los años 20, Universidad de Valladolid, Valladolid.

JIMÉNEZ MILLÁN, Antonio, (1984), Vanguardia e ideología. Aproximación a la historia de las literaturas de Vanguardia en Europa (1900-1930), Universidad de Málaga.

KHAN-MAGOMEDOV, Selim Omarovich, (1987), Pioneers of soviet architecture: the search for new solutions in the 1920 and 1930, Rizzoli, Nueva York,

LAVRIENTIEV, Aleksandr Nikolaievich, (2007), "Moskovskiy VKHUTEMAS - shkola konstruktivizma" en Istoriya dizayna, Gardariki, Moscú.

LODDER, Christina, (1988), El constructivismo ruso, Alianza, Madrid.

MIKHAYLOV, S.M. (2002), Istoriya dizayna. Vol. 1, Soyuz dizaynerov, Moscú.

KHAN-MAGOMEDOV, Selim Omarovich, (1990), Vhutemas: Moscou 1920 - 1930, Editions du Regard, Paris.

KORINA, Nataliya Dmitrevna, (2014), "Moskovskoe uchilische zhivopisi, vayaniya i zodchestva kak tsentr formirovaniya Moskovskoy shkoly zhivopisi v seredine XIX - nachala XX vekov.” en Vestnik PSTGU, serie V, Moscú.

TOCA FERNÁNDEZ, Antonio, (2016), "Una enseñanza revolucionaria: los VKHUTEMAS de Mosc 1920-1930” en Tiempo en Casa, núm.25, Universidad Autónoma Metropolitana, México.

ZHADOVA, Larisa Alekseievna, (1964), "VKHUTEMAS-VKHUTEIN" en Dekorativnoe iskusstvo SSSR, Nauka, Moscú.

VARST, "Rabochiy klub. Konstruktivist A.M. Rodchenko.” en Arquitectura Contemporánea, 1926, N1.

VVAA. (1970), Iz istorii sovetskoy arkhitektury. Dokumenty i materialy., Nauka, Moscú.

VVAA. (1927), Raboty arkhitekturnogo fakulteta VKHUTEMASa 1920- 1927, VKHUTEMAS,

Moscú. 


\section{Fuentes de internet:}

- Arzamas. Recuperado de: http://arzamas.academy/courses/18 (Consulta: 11/09/16)

- KHAN-MAGOMEDOV, S.O., Arkhitektura sovetskogo avangarda. Recuperado de: http://www.alyoshin.ru/Files/publika/khan_archi/khan_archi_1_000.ｈtml（Consulta: $13 / 05 / 16)$

- $\quad$ KLYUEV, M., Rossiyskoe napravlenie razvitiya dizayna: V K H U T E M A S. Recuperado de: http://www.gldesign.ru/index.php?catid=37:demo-52 content\&id=63:2009-05-06-21-3046\&Itemid=37\&option=com_content\&view=article $($ Consulta: $19 / 06 / 16)$

- $\quad$ KOYNOVA, N.V., Rol'VKHUTEMASa i Baukhauza v stanovlenii obrazovaniya v oblastyah promyshlennogo dizayna. Recuperado de: http://cyberleninka.ru/article/n/rol-vhutemasa-ibauhauza-v- stanovlenii-obrazovaniya-v-oblasti-promyshlennogo-dizayna (Consulta:3/06/16)

- KOYNOVA, N.V., Razvitie predstavleniya o forme $\mathrm{v}$ metodologii pervyh shkol dizayna VKHUTEMAS a i Baukhauza. Recuperado de: http://archvuz.ru/2011_22/62 (Consulta: $3 / 06 / 16)$

- Mobile Design Museum. Recuperado de: http://mobiledesignmuseum.ru/avangard/ (Consulta: 21/08/16)

- Afisha Daily, Prostranstvo VKHUTEMASa: gid po festivalyu. Recuperado de: https://daily.afisha.ru/archive/gorod/archive/8064/ (Consulta: 1/06/16)

- Recopilación de revistas de SA. Recuperado de: http://tehne.com/library/sovremennayaarhitektura-zhurnal-1926- 1930 (Consulta: 12/07/16)

- RARUS'S GALLERY. Recuperado de: http://www.raruss.ru/soviet- constructivism/3942soviet-constructivism.html?start=4 (Consulta: 10/09/16)

- Revista Togda. Recuperado de: http://www.togdazine.ru/tag/111 (Consulta: 15/08/16)

- $\quad$ VVAA., Ot VKHUTEMASa k MARKHi, 1920-1936., A-Fond, Moscú, 2005. Recuperado de: https://issuu.com/marhi/docs/v-m (Consulta: 22/06/16) 